\title{
An approach for solving multi-objective assignment problem with interval parameters
}

\author{
Kayvan Salehi*
}

Department of Industrial Engineering, Payam Noor University of Boukan, P. O. Box: 59516-79848, Boukan, Iran \section{H R O N I C L E}

Article history:

Received March 22014

Accepted 29 July 2014

Available online

July 312014

Keywords:

Assignment problem

Interval number

Weighted min-max method

Integer programming

\section{A B S T R A C T}

\begin{abstract}
This paper presents a multi-objective assignment problem (MAP) with interval parameters. Here, the model concentrates on three criteria: total cost, total profit and total operation time of assignment. The proposed model is classified as a nonlinear programming, in which it is difficult to solve. Hence, the model is converted into crisp linear programming problem by applying a substitution variables approach. In addition, a weighted min-max method is applied to transform the multi-objective problem into a single objective optimization problem. Finally, several numerical examples are provided to illustrate the implementation of the proposed approach. The results clearly provide some evidences that the proposed approach was easier to contrast compared with other approaches. The results also indicate that decision makers' preferences over various objectives could influence on solutions.
\end{abstract}

\section{Introduction}

Assignment problem (AP) is one of the most important issues in manufacturing and service systems. It has also received significant amount of attention among researchers. In AP, there are $N$ jobs that must be performed by $N$ workers, where the costs depend on the specific assignments. Each job must be assigned to one and only one worker and each worker has to perform one and only one job. The problem is to find such an assignment to minimize the total cost (Lin et al. 2011). An AP can be modeled as a $0-1$ integer programming as follows:

$$
\begin{cases}\min & f_{1}=\sum_{i=1}^{N} \sum_{j=1}^{N} c_{i j} x_{i j} \\ \text { s.t. } & \sum_{j=1}^{N} x_{i j}=1, \quad i=1,2, \ldots, N . \\ & \sum_{i=1}^{N} x_{i j}=1, \quad j=1,2, \ldots, N . \\ & x_{i j} \in\{0,1\}\end{cases}
$$

*Corresponding author. Tel: +98-938-1631744 
Here, $c_{i j}$ represents the cost associated with worker $i(i=1,2, \ldots, N)$ who has performed job $j$ $(j=1,2, \ldots, N)$. In classical AP model $(1)$ it is assumed that all the $c_{i j}$ 's are deterministic numbers. However, because of the existing uncertainty in real-world applications, costs are not known, exactly. Recently a number of researchers have investigated AP under random variables (Bogomolnaia, 2001; Bogomolnaia \& Moulin, 2002; Coppersmith \& Sorkin, 1999) and fuzzy variables (Bit et al. 1994; Belacela \& Boulasselb, 2001; Ridwan, 2004; Lin \& Wen, 2004; Liu \& Li, 2006; Feng \& Yang, 2006; Liu \& Gao, 2009; Mukherjee \& Basu, 2010; Mukherjee \& Basu, 2011; Kumar \& Gupta, 2012). Although, probability theory is one of the main tools used for analyzing uncertainty in the real world, however a great deal of knowledge about the statistical distribution of the uncertain parameters is required to define the parameters as random numbers with probability distributions. In addition, employing fuzzy theory to model uncertainty in real problems is criticized as using this type of approach and it is assumed that the membership functions of parameters are known. However, in reality, it is not always easy for a decision maker to specify it. In order to overcome this problem some researchers have applied interval programming. In really using interval programming does not require the specification or the assumption of probabilistic distribution (as in stochastic programming) or possibility distributions (as in fuzzy programming) (Zhou et al., 2009). Hence, it is a good idea for the decision makers to determine the uncertain as intervals. On the other hand, most of the studies for the AP have considered only one-objective function i.e. minimum cost. However, in AP models, the decision makers may consider several objectives together such as the minimum cost, maximum profit and the minimum operation time, which are more consistent with the decision making realities. There are few studies on multi-objective assignment problem (MAP) with interval parameters in literature. To the best of our knowledge, Kagade and Bajaj (2010) were first who proposed a MAP with interval parameters. They employed interval arithmetic for converting their model to crisp form. In their model right limit and center of the interval objective functions, are minimized. Hence, a problem with three objective functions converts to a deterministic programming with six objective functions. Hence, solving the model is time consuming. Therefore, the aim of this paper is threefold: First, we propose a (MAP) with interval parameters. Second, a new and easy approach is employed to transform this model into crisp form. Third, a weighted min-max method will use for solving the final model. To achieve this purpose, the rest of this paper is organized as

follows: In section 2, a (MAP) with interval parameters is explained and is converted to crisp form through a new approach. Section 3 proposes a weighted min-max method to transform multiobjective programming to single objective programming. In order to evaluate efficiency proposed model, two numerical examples is given in section 4. Finally, in Section 5, we draw conclusions and future researches.

\section{Multi-objective assignment problem with interval parameters}

In AP models, the decision makers may consider objectives in the frame of multi-objective problems, which are more consistent with the decision making realities. In addition to, due to existence of uncertainty in real applications, the models' parameters such cost, profit are not available, completely. Considering the uncertainty as interval numbers, the proposed MAP with interval parameters in this paper can be given as follows:

$$
\left\{\begin{array}{l}
\min f_{1}=\sum_{i=1}^{N} \sum_{j=1}^{N}\left[c_{i j}^{L}, c_{i j}^{R}\right] x_{i j} \\
\max f_{2}=\sum_{i=1}^{N} \sum_{j=1}^{N}\left[p_{i j}^{L}, p_{i j}^{R}\right] x_{i j} \\
\min f_{3}=\sum_{i=1}^{N} \sum_{j=1}^{N}\left[t_{i j}^{L}, t_{i j}^{R}\right] x_{i j} \\
\text { s.t. } \sum_{j=1}^{N} x_{i j}=1, i=1,2, \ldots, N, \quad \sum_{i=1}^{N} x_{i j}=1, j=1,2, \ldots, N, x_{i j} \in\{0,1\}
\end{array}\right.
$$


The first, second and three objectives describe the operation cost, operating profit and operating time objective function, respectively. First objective function focuses on how to assign jobs to workers so that the total operating cost can be minimized. Second objective function is associated with total operating profit maximization. In addition, the third objective function focuses on how to assign the jobs to workers so that the total operation time is minimized.

\subsection{Transformation approach}

In this section, an approach is proposed for transforming MAP to multi-objective linear programming (MLP). The approach is similar to Saati et al. (2002). By substituting the new variables, model (2) can be written as follows:

$$
\left\{\begin{array}{l}
\min f_{1}=\sum_{i=1}^{N} \sum_{j=1}^{N} c_{i j} x_{i j} \\
\max f_{2}=\sum_{i=1}^{N} \sum_{j=1}^{N} p_{i j} x_{i j} \\
\min f_{3}=\sum_{i=1}^{N} \sum_{j=1}^{N} t_{i j} x_{i j} \\
\text { s.t. } \sum_{j=1}^{N} x_{i j}=1, i=1,2, \ldots, N . \\
\quad \sum_{i=1}^{N} x_{i j}=1, j=1,2, \ldots, N . \\
c_{i j}^{L} \leq c_{i j} \leq c_{i j}^{R} \quad p_{i j}^{L} \leq p_{i j} \leq p_{i j}^{R} \quad t_{i j}^{L} \leq t_{i j} \leq t_{i j}^{R}, \quad x_{i j} \in\{0,1\}
\end{array}\right.
$$

The model (3) is a multi-objective nonlinear programming. Using the other substitution variables the model (3) is linearized as follows:

$$
\left\{\begin{array}{l}
\min f_{1}=\sum_{i=1}^{N} \sum_{j=1}^{N} \bar{c}_{i j} \\
\max f_{2}=\sum_{i=1}^{N} \sum_{j=1}^{N} \bar{p}_{i j} \\
\min f_{3}=\sum_{i=1}^{N} \sum_{j=1}^{N} \bar{t}_{i j} \\
\text { s.t. } \sum_{j=1}^{N} x_{i j}=1, i=1,2, \ldots, N . \\
\quad \sum_{i=1}^{N} x_{i j}=1, j=1,2, \ldots, N . \\
\quad x_{i j} c_{i j}^{L} \leq \bar{c}_{i j} \leq c_{i j}^{R} x_{i j} \quad x_{i j} p_{i j}^{L} \leq \bar{p}_{i j} \leq p_{i j}^{R} x_{i j} \quad x_{i j} t_{i j}^{L} \leq \bar{t}_{i j} \leq t_{i j}^{R} x_{i j}, \quad x_{i j} \in\{0,1\}
\end{array}\right.
$$

The model (4) is a multi-objective linear programming, where it is partly simple to solve in contrast with the models (2) and (3). In the next section, we present an efficient method for handling this model.

\section{Solution approach}

The extended model given in Eq. (4) is a multi-objective programming. There are several approaches for handling multi-objective optimization problems such as global criteria methods, compromise solutions (CP), scalarization, etc. In this paper, the weighted min-max method is applied to convert the multi-objective problem into a single objective optimization problem (Marler \& Arora, 2004). Before presenting the proposed approach consider the following multi-objective programming. The general multi-objective optimization problem is posed as follows: 


$$
\begin{aligned}
& \min _{x}^{2158} F(x)=\left[F_{1}(x), F_{2}(x), F_{3}(x), \ldots ., F_{k}(x)\right] \\
& \text { s.t.: } g_{j}(x) \leq 0, \mathrm{j}=1,2,3, \ldots, \mathrm{m}, \quad \mathrm{h}_{i}(x)=0, i=1,2,3, \ldots e .
\end{aligned}
$$

where $k$ is the number of objective functions, $m$ is the number of inequality constraints, and $e$ is the number of equality constraints. Therefore, the weighted min-max formulation, or weighted Tchebycheff method, is given as follows:

$U=\max _{i}\left\{w_{i}\left[F_{i}\left(x_{i}\right)-F_{i}^{o}\right]\right\}$.

Consequently, Eq. (6) can be rewritten as follows:

$\min \lambda$

s.t. $\quad w_{i}\left[F_{i}(x)-F_{i}^{o}\right]-\lambda \leq 0 \quad i=1,2,3, \ldots, k$.

The advantages of the method are as follows (Marler \& Arora, 2004):

- It provides an interpretation of the minimization of the largest difference between $F_{i}\left(x_{i}\right)$ and $F_{i}^{o}$,

- It can provide all of Pareto optimal points,

- It always provides a weakly Pareto optimal solution,

- It is relatively well suited for generating the complete Pareto optimal set.

The disadvantages are as follows (Marler \& Arora, 2004):

- It requires the minimization of the objective when using the utopia point.

- It requires that additional constraints be included.

- It is not clear exactly how to set the weight when only one solution point is desired.

Therefore, the model given in Eq. (4) can be written as follows:

$$
\left\{\begin{array}{l}
\min \quad \lambda \\
w_{1}\left[f_{1}-f_{1}^{o}\right]-\lambda \leq 0 \quad w_{2}\left[f_{2}-f_{2}^{o}\right]-\lambda \leq 0 \quad w_{3}\left[f_{3}-f_{3}^{o}\right]-\lambda \leq 0 \\
\text { where: } \\
\quad f_{1}=\sum_{i=1}^{N} \sum_{j=1}^{N} \bar{c}_{i j} \quad f_{2}=\sum_{i=1}^{N} \sum_{j=1}^{N} \bar{p}_{i j} \quad f_{3}=\sum_{i=1}^{N} \sum_{j=1}^{N} \bar{t}_{i j} \\
\text { s.t. } \sum_{j=1}^{N} x_{i j}=1, i=1,2, \ldots, N . \\
\quad \sum_{i=1}^{N} x_{i j}=1, j=1,2, \ldots, N . \\
\quad x_{i j} c_{i j}^{L} \leq \bar{c}_{i j} \leq c_{i j}^{R} x_{i j} \quad x_{i j} p_{i j}^{L} \leq \bar{p}_{i j} \leq p_{i j}^{R} x_{i j} \quad x_{i j} t_{i j}^{L} \leq \bar{t}_{i j} \leq t_{i j}^{R} x_{i j}, \quad x_{i j} \in\{0,1\}
\end{array}\right.
$$

where $w_{1}, w_{2}$ and $w_{3}$ are the weighting coefficients and provide the Pareto optimal solutions of the original problem presented in Eq. (4). A point $x^{*} \in R^{n}$ is called a Pareto optimal solution or noninferior solution to an optimization problem if there is no $x \in R^{n}$ satisfying $f_{i}(x) \leq f_{i}\left(x^{*}\right),(i=1,2 \ldots$, $n)$. In other words, $x^{*}$ is Pareto optimal if there exists no feasible vector of decision variables in the search space which would decrease some objectives without causing an increase in at least one other objective simultaneously (Zhou et al., 2009).

\section{Numerical examples}

In order to illustrate efficiency the proposed approach two numerical examples is provided. These examples are performed on a personal computer, 2.6 GHz CPU, 512MB memory. 
Example 1: In this example, it is assumed that the number of jobs is 3 and the number of workers (machine) is 3. This example is originally given in Kagade and Bajaj (2010). The cost matrixes are given as follows:

$$
c^{1}=\left(\begin{array}{ccc}
{[1,3]} & {[5,9]} & {[4,8]} \\
{[7,10]} & {[2,6]} & {[3,5]} \\
{[7,11]} & {[3,5]} & {[5,7]}
\end{array}\right) \quad c^{2}=\left(\begin{array}{ccc}
{[3,5]} & {[2,4]} & {[1,5]} \\
{[4,6]} & {[7,10]} & {[9,11]} \\
{[4,8]} & {[3,6]} & {[1,2]}
\end{array}\right)
$$

The problem was solved by the LINGO Software and Table 1 summarizes the results.

Table 1

Example 1

\begin{tabular}{llll}
\hline The weights & The obtained results & The weights & The obtained results \\
\hline $\mathrm{W}=(0.2,0.8)$ & $x^{*}=\left\{x_{12}=x_{21}=x_{33}=1\right\}$ & $\mathrm{W}=(0.8,0.2)$ & $x^{*}=\left\{x_{11}=x_{23}=x_{32}=1\right\}$ \\
$\mathrm{W}=(0.5,0.5)$ & $x^{*}=\left\{x_{11}=x_{22}=x_{33}=1\right\}$ \\
\hline
\end{tabular}

Kagade and Bajaj (2010) considered equal weights for objective functions. The proposed approach obtains the same results for the equal weights. However, our approach is very easy in contrast to their approach. In addition, the results show that decision makers' preferences over various objectives influence directly on the results. Notethat the results for many of the weights is equal.

Example 2: Now assume that the number of jobs is 3 and the number of workers (machine) is 3 . The operating cost, operating profit and operating time matrixes are given as follows:

$$
c=\left(\begin{array}{lll}
{[2,4]} & {[1,3]} & {[3,6]} \\
{[3,4]} & {[3,5]} & {[2,3]} \\
{[6,9]} & {[1,4]} & {[3,7]}
\end{array}\right) \quad p=\left(\begin{array}{lll}
{[3,6]} & {[2,4]} & {[1,3]} \\
{[7,9]} & {[3,6]} & {[4,6]} \\
{[1,4]} & {[3,5]} & {[2,5]}
\end{array}\right) \quad t=\left(\begin{array}{ccc}
{[5,8]} & {[1,3]} & {[4,7]} \\
{[3,7]} & {[7,10]} & {[5,8]} \\
{[3,6]} & {[1,3]} & {[5,8]}
\end{array}\right)
$$

The problem was solved by the LINGO Software and Table 2 shows the results.

Table 2

Example 2

\begin{tabular}{rlll}
\hline The weights & The obtained results & The weights & The obtained results \\
\hline $\mathrm{W}=(1 / 3,1 / 3,1 / 3)$ & $x^{*}=\left\{x_{13}=x_{21}=x_{32}=1\right\}$ & $\mathrm{W}=(0.5,0.3,0.2)$ & $x^{*}=\left\{x_{12}=x_{23}=x_{31}=1\right\}$ \\
$\mathrm{W}=(0.7,0.2,0.1)$ & $x^{*}=\left\{x_{11}=x_{23}=x_{32}=1\right\}$ \\
\hline
\end{tabular}

The results of the examples 1 and 2 show that decision maker' preferences over various objectives could influence on the results. Therefore, it is essential for the decision makers to have a logical decision on the weighting of the various objectives, since improper preferences over objectives may lead to have an improper solutions. Here in the qualitative methods such as brainstorming or Delphi method can be used. Moreover, the quantity approaches such as analytical hierarchy process (AHP) can be employed to prioritization the objectives.

\section{Summary and conclusions}

In this paper, we proposed a multi-objective assignment problem (MAP) by considering models' parameters as interval numbers. The proposed model was converted into crisp form through substation variables. The preliminary results of solving the model by weighted min-max method showed that the proposed approach could yield promising results. In contrast to Kagade and Bajaj 
(2010) model, which convert the AP model to four linear programming to obtain optimal solutions in a two objective problem, our method solves a two linear programming problem. In addition, our method is very easy in contrast to their approach, since it would not need for employing interval computation in interval programming. In addition, the results indicated that decision makers' preferences over various objectives could influence on solutions. Further research will focus on using the other methods to solve AP models. In addition, the proposed approach in this paper can be used in the other problems in the field of industrial engineering and management sciences.

\section{References}

Belacela, N., \& Boulasselb, M.R. (2001). Multicriteria fuzzy assignment method: a useful tool to assist medical diagnosis. Artificial Intelligence Medicine, 21, 201-207.

Bit, A.K., Biswal, M.P., \& Alam, S.S. (1994). Fuzzy programming approach to multi-objective assignment problem. Journal of Fuzzy Mathematics (USA), 2, 905-909.

Bogomolnaia, A. (2001). A new solution to the random assignment problem. Journal of Economic Theory, 100, 295-328.

Bogomolnaia, A., \& Moulin, H. (2002). A simple random assignment problem with a unique solution. Economic Theory, 19(3), 623-636.

Coppersmith, D., \& Sorkin, G.B. (1999). Constructive bounds and exact expectation for the random assignment problem. Random Structure Algorithm, 15(2), 113-144.

Feng, Y., \& Yang, L. (2006). A two-objective fuzzy k-cardinality assignment problem. Journal of Computational Applied Mathematics, 197, 233-244.

Kagade, K. L., \& Bajaj, V. H. (2010). Fuzzy method for solving multi-objective assignment problem with interval cost. Journal of Statistics and Mathematics, 1, 01-09.

Kumar, A. and Gupta, A. (2012). Assignment and Travelling Salesman Problems with Coefficients as LR Fuzzy Parameters. International Journal of Applied Science and Engineering, 10(3), 155-170.

Lin, C.J., \& Wen, U.P. (2004). The labeling algorithm for the fuzzy assignment problem. Fuzzy Set and Systems, 142, 373-391.

Lin,C.J., Wen, U.P., \& Lin, P.Y. (2011). Advanced sensitivity analysis of the fuzzy assignment problem. Applied Soft Computation, 11, 5341-5349

Liu, L., \& Gao, X. (2009). Fuzzy weighted equilibrium multi-job assignment problem and genetic algorithm. Applied Mathematical Modeling, 33, 3926-3935.

Liu, L., \& Li, Y. (2006). The fuzzy quadratic assignment problem with penalty: new models and genetic algorithm. Applied Mathematical Computation, 174, 1229-1244.

Marler, R. T., \& Arora, J. S. (2004). Survey of multi-objective optimization methods for engineering. Structural and multidisciplinary optimization, 26(6), 369-395.

Mukherjee, S., \& Basu, K. (2010). Application of fuzzy ranking method for solving assignment problems with fuzzy costs. International Journal of Computational and Applied Mathematics, 5, 359-368.

Mukherjee, S., \& Basu, K. (2011). Solving intuitionistic fuzzy assignment problem by using similarity measures and score functions. International Journal of Pure and Applied Sciences and Technology, 2(1), 1-18.

Ridwan, M. (2004). Fuzzy preference based traffic assignment problem. Transportation Research Part C, 12, 209-233.

Saati, S., Memariani, A., \& Jahanshahloo, G.R. (2002). Efficiency analysis and ranking of decision making units with fuzzy data. Fuzzy Optimization and Decision Making, 1(3), 255-267.

Zhou, F., Huang, G.H., Chen, G.X., \& Guo, H.C. (2009). Enhanced-interval linear programming. European Journal of Operational Research, 199, 323-333 